\title{
Pulmonary Endocarditis with Spondylodiscitis: A lucky Finding
}

\author{
Randa Tabbah ${ }^{1 *}$, Rachoin Rachoin ${ }^{2}$ and Edouard Elias ${ }^{3}$ \\ ${ }^{1}$ Department of cardiology, Notre Dame des Secours Jbeil (CHUNDS), Holy spirit of Kaslik, Beirut, Lebanon
}

${ }^{2}$ Department of cardiology, Head of echocardiography, Notre Dame des Secours Jbeil (CHUNDS), Hotel Dieu de France, Holy spirit of kaslik, Beirut

${ }^{3}$ Head of department of Internal medicine, Notre Dame des Secours Jbeil (CHUNDS), Holy spirit of Kaslik, Beirut, Lebanon

Corresponding author: Randa Tabbah, Department of cardiology, Notre Dame des Secours Jbeil (CHUNDS), Holy spirit of Kaslik,

Beirut, Lebanon

\section{ARTICLE INFO}

Received: 唪 October 01, 2020

Published: 蔧 October 20, 2020

Citation: Randa Tabbah, Rachoin Rachoin, Edouard Elias. Pulmonary Endocarditis with Spondylodiscitis: A lucky Finding. Biomed J Sci \& Tech Res 31(2)-2020. BJSTR. MS.ID.005079.

\section{ABSTRACT}

Spondylodiscitis and endocarditis is a rare association especially when involving a pulmonary endocarditis. We describe a case of a 61 year old male patient presented to his primary physician for back pain for more than 3 months. His history revealed an episode of community acquired pneumonia 4 months before his presentation to the clinic. Patient still complaining about a nonproductive cough since his treatment. His physical examination revealed a murmur on the left lateral sternal border. Patient report pain on percussion over the spinal segment. Laboratory tests revealed normal blood count with normal white blood cells and normal inflammatory parameters. Blood cultures were negative. MRI of the spine showed erosions of the vertebral plates in L4 and L5 and an edematous regional infiltration of the psoas muscle are common signs of spondylodiscitis with an anterior epidural abscess. Cardiac ultrasound revealed a moderate pulmonary regurgitation grade II with a hyperechogenic image on the pulmonary valve measuring $(14 \times 20 \mathrm{~mm})$ suspicious of a vegetation. TEE showed signs of a pulmonary vegetation with a moderate pulmonary regurgitation and no signs of abscess. Treatment with antibiotics for 6 weeks was initiated. Cardiac ultrasound after regimen revealed stable vegetation more hyperechogenic and calcified as a sign of chronic endocarditis. His last MRI revealed complete regression of the epidural collection with residual signs of spondylodiscitis. This case highlights the diagnostic challenge of characterizing pulmonary valve endocarditis and regurgitation. It also emphasizes on the management challenges of these rare cases that need to be adjusted. Close monitoring is mandatory and regular cardiac ultrasound is the key for diagnosis and follow up.

\section{Introduction}

In 1965, Sèze et al described a first case associating spondylodiscitis and endocarditis [1]. Since then many case reports outlined this combination but it is controversial and prospective studies still lacking [2-11]. The frequency of this association is ranging from $0.6 \%-2.2 \%$, when patients with established spondylodiscitis were screened for endocarditis [2,3]. Musculoskeletal symptoms were associated with this combination [12]. We describe a rare case of pulmonary endocarditis a very rare finding in a patient presented for spondylodiscitis.

\section{Clinical Case}

A 61 year old male patient presented to his primary physician for back pain for more than 3 months. His history revealed no previous medical issues, but the patient mentioned an episode of community acquired pneumonia 4 months before his presentation to the clinic. He noticed the need for intravenous antibiotics for 2 weeks. In addition, patient still complaining from a nonproductive cough since his treatment. He had no fever nor night sweats but 
complained of fatigue and arthralgia. On physical examination, lungs were clear, no pitting edema, normal heart sounds although a murmur was heard on the left lateral sternal border. Patient report pain on percussion over the spinal segment but no or little pain on pressure. Inclining and straightening up again causes pain to increase. Pain on knee flexion was also seen. Upon exertion pain also increases. Laboratory tests revealed normal blood count with normal white blood cells and normal inflammatory parameters
(CRP, ESR, Procalcitonin.). Blood cultures were negative. MRI of the spine showed erosions of the vertebral plates in L4 and L5 with epidural thickening pushing back the dural sac with peripheral enhancement and hypointense center of the intervertebral disc of L4 and L5 in favor of an epidural abscess.Furthermore, an edematous regional infiltration of the psoas muscle are common signs of spondylodiscitis with an anterior epidural abscess (Figure $1 \mathrm{~A})$.

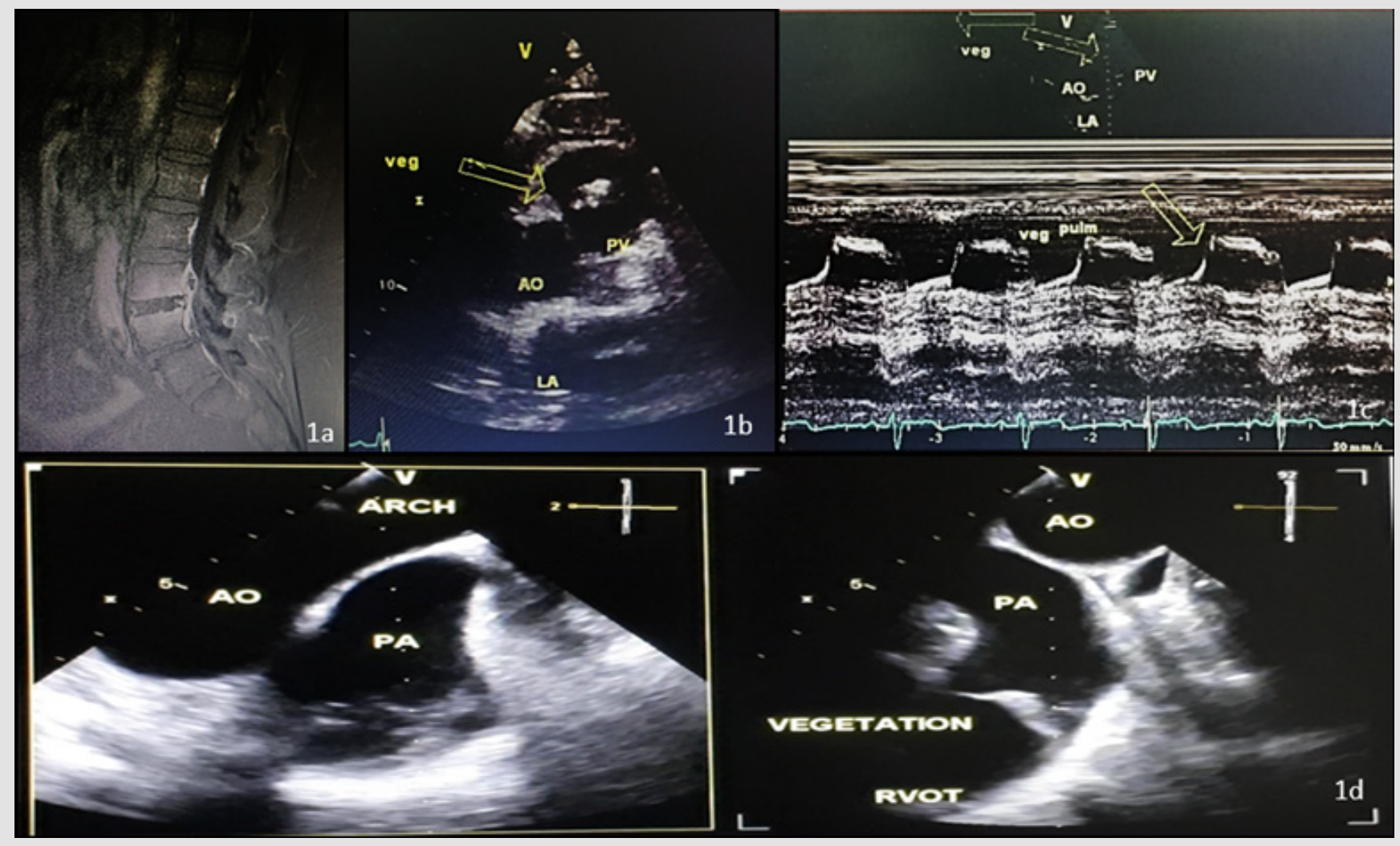

Figure 1A: MRI of the spine revealing an epidural abscess with signs of spondylodiscitis .

Figure 1B: Transthoracic echocardiography (short axis) revealing a hyperechogenic image on the pulmonary valve measuring $(14 \times 20 \mathrm{~mm})$ suspicious of a vegetation.

Figure 1C: Transthoracic echocardiography, signs of vegetation on TM .

Figure 1D: Transesophageal echocardiography with a view of the pulmonary valve and signs of vegetation with a moderate pulmonary regurgitation.

Cardiac ultrasound revealed a preserved Left ventricular function with no indirect signs of pulmonary hypertension, normal diastolic function and left ventricular end diastolic pressure with an aneurysmal interatrial septum. Aortic and mitral valve were normal but there was a moderate pulmonary regurgitation grade II with a hyperechogenic image on the pulmonary valve measuring $(14 \times 20 \mathrm{~mm})$ suspicious of a vegetation. (Figure $1 \mathrm{~B} \&$ 1C)TEE showed signs of a pulmonary vegetation with a moderate pulmonary regurgitation and no signs of abscess [Figure1d]. Treatment with antibiotics for 6 weeks was initiated. Cardiac ultrasound after regimen revealed stable vegetation more hyperechogenic and calcified as a sign of chronic endocarditis. His last MRI revealed complete regression of the epidural collection with residual signs of spondylodiscitis. Patient stable, pain is better and follow up with cardiac ultrasound every 6 months is mandatory. No signs of right ventricular dysfunction were noticed and pulmonary regurgitation still stable. Patient feeling better.

\section{Discussion}

Pulmonic Valve (PV) Infective Endocarditis (IE) is a rare finding, accounting for $1.5 \%$ to $2 \%$ of the cases of endocarditis. It is an extremely rare disease that involving both normal 
and congenital valves. This condition occurs in patients with underlying predisposingfactors Intravenous (IV) drug abuse the most common, male gender, central venous catheters, alcoholism, dental extraction, gonorrhea, liver or renal transplantation, bowel surgery,colonic angiodysplasia, and congenital heart disease). In our case no specific risk factor for pulmonary endocarditis were found [13,14]. The pathogenesis of spondylodiscitis associated to infectious endocarditis is a dilemma, that relies on different hypothesis. A hematogenous dissemination of the infection agent orvertebral deposition of arterial emboli that could be bacterial or also immunological[15]. Although the prognosis of the patient depends mainly on the valvular disease, appropriate determination of this association the need of a longer antibiotic course is important and can range from 6 weeks to 3 months as in our case it was 6 weeks of treatment [12]. We conclude that the association of spondylodiscitis and endocarditis should always be considered in the setting of musculoskeletal symptoms even pulmonary endocarditis despite in the absence of classical risk factors as in this case.

\section{Conclusion}

In conclusion, pulmonic valve endocarditis is an extremely rare infection that could be missed if patient presentation is atypical with no specific risk factors nor typical features ofright-sided endocarditis. To our knowledge, this is an extremely rare finding in an asymptomatic patient with no fever nor elevated inflammatory labs associated with a spondylodiscitis and presenting mainly for back pain. This case highlights the diagnostic challenge of characterizing pulmonary valve endocarditis and regurgitation. It also emphasizes on the management challenges of these rare cases that need to be adjusted. Close monitoring is mandatory and regular cardiac ultrasound is the key for diagnosis and follow up.

\section{References}

1. Sèze S de, Ryckewaert A, Kahn MF (1965) L endocardite d Osler em rhumatologie. Rev Rhum Mal Ostéoartic 32: 739-44.
2. Meys E, Deprez X, Hautefeuille P (1991) Place des spondylodiscites iatrogènes parmi lês spondylodiscites à germes banals. Rev Rhum Mal Ostéoartic 58: 839-846.

3. Hacène A, Flipo RM, Deprez X (1996) Endocardites et spondylodiscites infectieuses. Rev Rhum 63: 742.

4. Le Moal G, Roblot F, Paccalin M (2002) Clinical and laboratory characteristics of infective endocarditis when associated with spondylodiscitis. Eur J Clin Microbiol Infect Dis 21: 671-675.

5. Morelli S, Carmenini E, Caporossi A (2001) Spondylodiscitis and infective endocarditis: case studies and review of the literature. Spine 26: 499-500.

6. Calderaro D, Galvão TF, Bagnatori RS, Caramelli B (2003) Spondilodiscitis as a clinical manifestation of infective endocarditis. Arq Bras Cardiol 81(5): 526-527.

7. Weber M, Gubler J, Fahrer H (1999) Spondylodiscitis caused by viridans streptococci: three cases and a review of the literature. Clin Rheumatol 18(5): 417-421.

8. Genta PR, Carneiro L, Genta EN (1998) Spreptococcus bovis bacteremia: unusual complications. South Med J 91(12): 1167-1168.

9. Ninet J, Gayet HL, Etienne J (1984) Bacterial endocarditis presenting as acute vertebral osteomyelitis: 14 cases. Eur Heart J 5(suppl C9): 101105.

10. McHenry MC, Easley KA, Locker GA (2002) Vertebral osteomyelitis:long term outcome for 253 patients from 7 Cleveland-area hospitals. Clin Infect Dis 34: 1342-1350.

11. Cusmano F, Calabrese G, Bassi S (2000) Radiologic diagnosis of spondylodiscitis: role of magnetic resonance. Radiol med (Torino) 100(3): 112-119.

12. Daniela Calderaro, Danielle Menosi Gualandro, Pai Ching Yu, Andre Coelho Marques, Luiz Boro Puig, Bruno Caramelli (2008) Spondylodiscitis and infectious endocarditis: a round-trip to be considered Braz J Infect Dis 12(6): 544-545.

13. Ramadan FB, Beanlands DS, Burwash IG (2000) Isolated pulmonic valve endocarditis in healthy hearts: a case report and review of the literature. Can J Cardiol 16(10): 1282-1288.

14. Cassling RS, Rogler WC, McManus BM (1985) Isolated pulmonic valve infective endocarditis: a diagnostically elusive entity. Am Heart J 109(3 Pt1): 558-567.

15. Lepeschkin E (1952) On the relation between the site of valvular involvement in endocarditis and the blood pressure resting on the valve. Am J Med Sci 224(3): 318-319.

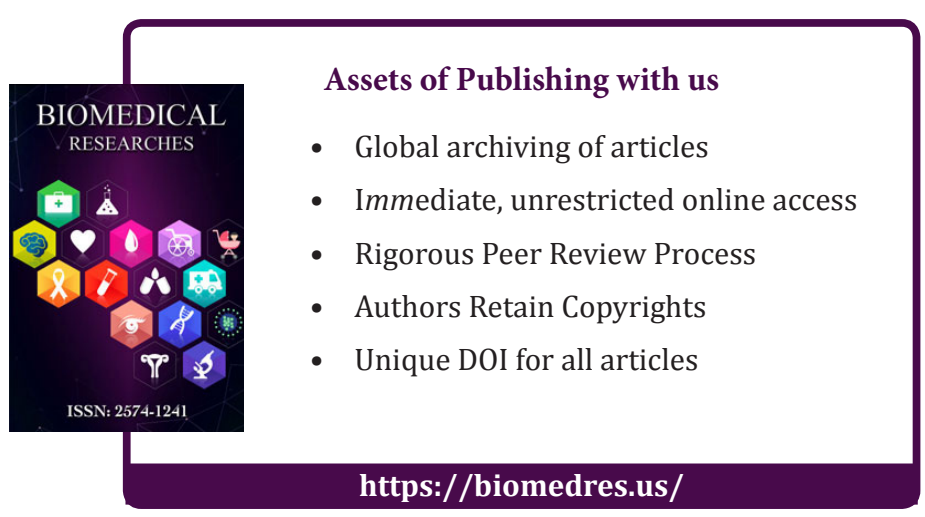

